\title{
LOCAL SUPREMA OF DIRICHLET POLYNOMIALS AND ZEROFREE REGIONS OF THE RIEMANN ZETA-FUNCTION
}

\author{
MICHEL J. G. WEBER \\ IRMA, 10 rue du Général Zimmer, 67084 Strasbourg Cedex, France \\ e-mail:michel.weber@math.unistra.fr
}

(Received 21 November 2012; revised 23 April 2013; accepted 31 July 2013)

\begin{abstract}
A new family of zerofree region of the Riemann Zeta-function $\zeta$ is identified by using Turán's (P. Turán, Eine neue Methode inter Analysis und deren Anwendungen (Akadémiai Kiadó, Budapest, Hungary, 1953); Analytic number theory, Proc. Symp. Pure Math., vol. XXIV (Amer. Math. Soc. Providence, RI, 1972)) localization criterion linking zeros of $\zeta$ with uniform local suprema of sets of Dirichlet polynomials expanded over the primes. The proof is based on a randomization argument. An estimate for local extrema for some finite families of shifted Dirichlet polynomials is established by preliminary considering their local increment properties by means of Montgomery-Vaughan's variant of Hilbert's inequality. A covering argument combined with Turán's localization criterion allows to conclude.
\end{abstract}

2010 Mathematics Subject Classification. Primary: 11M26; Secondary: 26D05, $60 \mathrm{G} 17$.

1. Main Result. The question of the existence of an eventual explicit relation between the zeros of the Riemann Zeta function $\zeta(s), s=\sigma+i t$ and the prime numbers was raised by Landau in [1]. Motivated by Landau's remark, Turán [3, 4, Chapters 33-36] had much investigated the connection between zerofree regions of $\zeta$ and local bounds of Dirichlet polynomials expanded over the primes. Among the several strong localization results stated in [4], the following semi-global criterion (Theorem 3') is of particular relevance in the present work.

Turán's Localization Criterion. Let $D$ be some positive real and $0<E \leq 9 / 10$. Suppose there exist positive reals $T, \beta, 0<\beta<1$ such that for $T-T^{E} \leq \tau \leq T+T^{E}$, the inequality

$$
\left|\sum_{N_{1} \leq p \leq N_{2}} p^{-i \tau}\right| \leq c \frac{N \log ^{10} N}{\tau^{\beta}}
$$

holds for

$$
T^{D\left(1-\beta^{1 / 6}\right)} \leq N \leq N_{1}<N_{2} \leq 2 N \leq T^{D\left(1+\beta^{1 / 6}\right)}
$$

where $c$ stands for positive numerical, explicitely calculable constant.

Then $\zeta(s) \neq 0$ in the parallelogram $\sigma>1-\beta^{2}, T-T^{E} \leq t \leq T+T^{E}$. 
In this paper, we show by using a local randomization argument that Turán's [3, 4] approach for localizing zeros of $\zeta$ is sufficiently powerful to permit to identify a completely new semi-global zerofree region.

Our main result states the following:

TheOREM 1.1. Let $0<\alpha^{*}<1$. There exist $1 / 2<\sigma_{0}<1, B \geq 4, v_{0}<\infty$, such that: For all $v \geq v_{0}$, there exists at least $\alpha^{*} 2^{B v+1}$ indices $j$ for which

$$
\zeta(\sigma+i t) \neq 0 \quad \forall \sigma \geq \sigma_{0}, \quad \forall t \in\left[2^{2 B v}+(j-1) 2^{B v-1}, 2^{2 B v}+j 2^{B v-1}[.\right.
$$

It follows from the proof that any value $\sigma_{0}>1-1 /(19)^{12}$ is, for instance, suitable. The same approach permits to get only slightly better thresholds.

In order to bound $\left|\sum_{N_{1} \leq p \leq N_{2}} p^{-i \tau}\right|$ uniformly over a family of suitable segments $\left[N_{1}, N_{2}\right]$ of the real line, we use an approach which can be described as follows. Let $\varphi_{1}, \ldots, \varphi_{N}$ be distinct reals. Consider a finite family of Dirichlet polynomials $P^{s}(t)=$ $\sum_{n=1}^{N} c_{n}^{S} e^{i t \varphi_{n}}, s \in S, c_{1}^{s}, \ldots, c_{N}^{s}$ being complex numbers. Instead of directly searching a bound of $\sup _{S}\left|P^{s}(t)\right|$ uniformly in $t$ over some finite interval $L$, we operate with the shifted Dirichlet polynomials,

$$
P_{\theta}^{s}(t)=\sum_{n=1}^{N} c_{n}^{s} e^{i(\theta+t) \varphi_{n}},
$$

where $\theta$ belongs to some fixed interval $J$. Given some interval $L,\left\{P_{\theta}^{s}(t), s \in S, t \in\right.$ $L, \theta \in J\}$ is up to some extent interpreted at an intermediate stage of the proof as a random process built on $J$ ( $\theta$ being treated as a random parameter), of which we estimate the increments by means of variant form of Hilbert's inequality due to Montgomery and Vaughan, and next control suprema, namely here $\sup _{t \in L} \sup _{S}\left|P_{\theta}^{s}(t)\right|$, by using its smoothness properties.

Another step is devoted to carefully adjusting some inherent family of parameters to apply Turán's $[3,4]$ result. Once this is achieved, a family of intervals $\left(I_{\theta}\right)_{\theta}$ free of zeros is exhibited. The family is indexed by a measurable set of $\theta$ 's of controlable positive measure. Finally, a covering argument allows to establish the existence of a semi-global region. This is the strategy we apply.

2. Local Mean Value Results. In the sequel, the parameter $s$ disappears since coefficients $c_{1}^{s}, \ldots, c_{N}^{s}$ used are simple subsets from a fixed set defined later on. Let $q$ be some positive integer and denote

$$
E_{q}=\left\{\underline{k}=\left(k_{1}, \ldots, k_{N}\right) ; k_{i} \in \mathbb{N} \cup\{0\}: k_{1}+\ldots+k_{N}=q\right\} .
$$

Let $\varphi_{1}, \ldots, \varphi_{N}$ be linearly independent reals. Introduce a coefficient of linear spacing of order $q$ by putting

$$
\xi_{\varphi}(N, q)=\inf _{\substack{h, k \in E_{q} \\ \underline{h} \neq \underline{\underline{k}}}}\left|\left(h_{1}-k_{1}\right) \varphi_{1}+\ldots+\left(h_{N}-k_{N}\right) \varphi_{N}\right| .
$$

By assumption $\xi_{\varphi}(N, q)>0$ and $\xi_{\varphi}(N, 1)=\inf \left\{\left|\varphi_{i}-\varphi_{j}\right|: i \neq j\right\}$. In the case $\varphi_{n}=$ $\log p_{n}, p_{n}$ denoting the $n$th consecutive prime, we have the classical estimate $\xi_{\varphi}(N, q) \geq$ $p_{N}^{-q}$, see proof before $(2.13)$. 
We estimate the local increments of $P$. defined in (1.2). Let $J$ be a bounded interval and let $|J|$ denote its length. Let $m_{J}$ denote the normalised Lebesgue measure on $J$. With the notation (1.2), if $J=[a, b]$ then $\|P .(t)-P .(s)\|_{m_{J}, 2 q}$ and $\|P .(t)\|_{m_{J}, 2 q}$ respectively denote

$$
\left(\frac{1}{b-a} \int_{a}^{b}|P(\theta+t)-P(\theta+s)|^{2 q} d \theta\right)^{1 / 2 q}, \quad\left(\frac{1}{b-a} \int_{a}^{b}|P(\theta+t)|^{2 q} d \theta\right)^{1 / 2 q} .
$$

Introduce the stationary metric on the real line defined by

$$
d(s, t)=d_{N}(s, t):=\left(2 \sum_{n=1}^{N}\left|c_{n}\right|^{2}\left|\sin \frac{(t-s) \varphi_{n}}{2}\right|^{2}\right)^{1 / 2}
$$

In the proposition below, $\xi$ is a simpler notation for $\xi_{\varphi}(N, q)$.

Proposition 2.1. (a) For any reals $s$ and $t$,

$$
\|P .(t)-P .(s)\|_{m_{J}, 2 q} \leq\left(q !+\frac{2 \min \left(N^{q}, \pi q !\right)}{|J| \xi}\right)^{1 / 2 q} d(s, t) ;
$$

and

$$
\|P .(t)\|_{m_{J}, 2 q} \leq\left(q !+\frac{2 \min \left(N^{q}, \pi q !\right)}{|J| \xi}\right)^{1 / 2 q}\left(\sum_{n=1}^{N}\left|c_{n}\right|^{2}\right)^{1 / 2}
$$

By taking $J=[-T, T], t=0$ in the last estimate, we deduce the following.

COROLLARY 2.2. We have the following bound

$$
\frac{1}{2 T} \int_{-T}^{T}\left|\sum_{n=1}^{N} c_{n} e^{i \theta \varphi_{n}}\right|^{2 q} d \theta \leq q !\left(1+\frac{2 \pi}{T \xi_{\varphi}(N, q)}\right)\left(\sum_{n=1}^{N}\left|c_{n}\right|^{2}\right)^{q} .
$$

In particular,

$$
\frac{1}{2 T} \int_{-T}^{T}\left|\sum_{p \leq N} \frac{c_{p}}{p^{i \theta}}\right|^{2 q} d \theta \leq q !\left(1+\frac{2 \pi N^{q}}{T}\right)\left(\sum_{p \leq N}\left|c_{p}\right|^{2}\right)^{q}
$$

Now put

$$
\mathcal{B}=\mathcal{B}_{\varphi}(J, N, q)=\left[q !\left(1+\frac{2 \pi}{|J| \xi_{\varphi}(N, q)}\right)\right]^{1 / 2 q}
$$

THEOREM 2.3. Let $\tilde{\varphi}_{N}=\sup _{n \leq N}\left|\varphi_{n}\right|$. There exists a constant $C_{q}$ depending on $q$ only such that for any interval $L$,

$$
\begin{aligned}
&\left\|\sup _{t \in L}|P .(t)|\right\|_{m_{J}, 2 q} \leq C_{q} \mathcal{B} \max \left\{1,|L| \tilde{\varphi}_{N}\right\}^{1 / 2 q}\left\{\left[\sum_{n=1}^{N}\left|c_{n}\right|^{2}\right]^{1 / 2}\right. \\
&\left.+\min \left(|L|, \frac{1}{\tilde{\varphi}_{N}}\right)\left[\sum_{n=1}^{N}\left|c_{n}\right|^{2} \varphi_{n}^{2}\right]^{1 / 2}\right\} .
\end{aligned}
$$


Proof of Proposition 2.1. Let $J=[d, d+T]$. Write more shortly $\xi=\xi_{\varphi}(N, q)$. Plainly,

$$
\begin{aligned}
(P(\theta+t)-P(\theta+s))^{q} & =\left(\sum_{n=1}^{N} c_{n} e^{i \theta \varphi_{n}}\left(e^{i t \varphi_{n}}-e^{i s \varphi_{n}}\right)\right)^{q} \\
& =\sum_{\underline{k} \in E_{q}} \frac{q !}{k_{1} ! \ldots k_{N} !} \prod_{n=1}^{N} c_{n}^{k_{n}} e^{i \theta k_{n} \varphi_{n}}\left(e^{i t \varphi_{n}}-e^{i s \varphi_{n}}\right)^{k_{n}} .
\end{aligned}
$$

Put $\gamma_{n}=e^{i t \varphi_{n}}-e^{i s \varphi_{n}}$. Thus,

$$
\begin{aligned}
\mid P & (\theta+t)-\left.P(\theta+s)\right|^{2 q} \\
& =\sum_{\underline{k}, \underline{h} \in E_{q}} \frac{(q !)^{2}}{k_{1} ! h_{1} ! \ldots k_{N} ! h_{N} !} \prod_{n=1}^{N} c_{n}^{k_{n}} \bar{c}_{n}^{h_{n}} e^{i \theta\left(k_{n}-h_{n}\right) \varphi_{n}} \gamma_{n}^{k_{n}} \bar{\gamma}_{n}^{h_{n}} \\
& =\sum_{\underline{k} \in E_{q}}\left(\frac{q !}{k_{1} ! \ldots k_{N} !}\right)^{2} \prod_{n=1}^{N}\left(\left|c_{n}\right|\left|\gamma_{n}\right|\right)^{2 k_{n}}+R(\theta),
\end{aligned}
$$

where

$$
R(\theta)=\sum_{\substack{k, h \in E_{q} \\ \underline{k} \neq \underline{\underline{n}}}}\left(\frac{(q !)^{2}}{k_{1} ! h_{1} ! \ldots k_{N} ! h_{N} !}\right) \prod_{n=1}^{N}\left(c_{n} \gamma_{n}\right)^{k_{n}}\left(\overline{c_{n} \gamma_{n}}\right)^{h_{n}} e^{i \theta\left(k_{n}-h_{n}\right) \varphi_{n}}
$$

Owing to linear independence, $\sum_{n=1}^{N}\left(k_{n}-h_{n}\right) \varphi_{n}=0$ iff $k_{n}=h_{n}, n=1, \ldots, N$. By integrating

$$
\begin{aligned}
& \frac{1}{T} \int_{J}|P(\theta+t)-P(\theta+s)|^{2 q} d \theta=\sum_{\underline{k} \in E_{q}}\left(\frac{q !}{k_{1} ! \ldots k_{N} !}\right)^{2} \prod_{n=1}^{N}\left(\left|c_{n}\right|\left|\gamma_{n}\right|\right)^{2 k_{n}} \\
& \quad+\sum_{\substack{k, h \in E_{q} \\
k \neq \underline{h}}} \frac{(q !)^{2}}{k_{1} ! h_{1} ! \ldots k_{N} ! h_{N} !} \prod_{n=1}^{N}\left(c_{n} \gamma_{n}\right)^{k_{n}}\left(\overline{c_{n} \gamma_{n}}\right)^{h_{n}} \\
& \quad \times\left[\frac{e^{i(d+T) \sum_{n=1}^{N}\left(k_{n}-h_{n}\right) \varphi_{n}}-e^{i d \sum_{n=1}^{N}\left(k_{n}-h_{n}\right) \varphi_{n}}}{i T\left(\sum_{n=1}^{N}\left(k_{n}-h_{n}\right) \varphi_{n}\right)}\right] .
\end{aligned}
$$

Put

$$
\mathbf{c}_{\underline{k}}=\prod_{n=1}^{N} \frac{\left(c_{n} \gamma_{n} e^{i(d+T) \varphi_{n}}\right)^{k_{n}}}{k_{n} !}, \quad \mathbf{d}_{\underline{k}}=\prod_{n=1}^{N} \frac{\left(c_{n} \gamma_{n} e^{i d \varphi_{n}}\right)^{k_{n}}}{k_{n} !}, \quad \mathbf{l}_{\underline{k}}=\sum_{n=1}^{N} k_{n} \varphi_{n} .
$$

Then

$$
\begin{aligned}
& \frac{1}{T} \int_{J}|P(\theta+t)-P(\theta+s)|^{2 q} d \theta \\
= & q !^{2} \sum_{\underline{k} \in E_{q}}\left|\mathbf{d}_{\underline{k}}\right|^{2}+\frac{(q !)^{2}}{i T}\left\{\sum_{\substack{\underline{k}, \underline{h} \in E_{q} \\
\underline{k} \neq \underline{\underline{k}}}} \frac{\mathbf{c}_{\underline{k}} \overline{\mathbf{c}}_{\underline{h}}}{\mathbf{l}_{\underline{k}}-\mathbf{l}_{\underline{h}}}-\sum_{\substack{\underline{k}, \underline{h} \in E_{q} \\
\underline{k} \neq \underline{h}}} \frac{\mathbf{d}_{\underline{k}} \overline{\mathbf{d}}_{\underline{h}}}{\mathbf{l}_{\underline{k}}-\mathbf{l}_{\underline{h}}}\right\} .
\end{aligned}
$$


Each of the two claimed bounds will now be deduced from either Hilbert's inequality or the Cauchy-Schwarz inequality. Recall Hilbert's inequality [2, p. 138]:

Let $\lambda_{1}, \ldots, \lambda_{N}$ be distinct real numbers, and suppose $\delta>0$ is chosen so that $\mid \lambda_{m}-$ $\lambda_{n} \mid \geq \delta$ whenever $n \neq m$. Then,

$$
\left|\sum_{\substack{1 \leq m, n \leq N \\ n \neq m}} \frac{x_{m} y_{n}}{\lambda_{m}-\lambda_{n}}\right| \leq \frac{\pi}{\delta}\left(\sum_{m=1}^{N}\left|x_{m}\right|^{2}\right)^{1 / 2}\left(\sum_{n=1}^{N}\left|y_{n}\right|^{2}\right)^{1 / 2} .
$$

We shall apply it under the following form: Let $\left\{x_{\underline{k}}, y_{\underline{k}}, \underline{k} \in E_{q}\right\}$. Also, let $\left\{\lambda_{\underline{k}}, \underline{k} \in E_{q}\right\}$ be distinct real numbers such that $\min \left\{\left|\lambda_{\underline{k}}-\lambda_{\underline{h}}\right|, \underline{k} \neq \underline{h}\right\} \geq \delta$. Let $v=\#\left\{E_{q}\right\}$ and consider a bijection $i:\{1, \ldots, v\} \rightarrow E_{q}$. By using $(2 . \overline{5})$

$$
\begin{aligned}
\left|\sum_{\substack{\underline{k}, \underline{h} \in E_{q} \\
\underline{\underline{k}} \neq \underline{\underline{k}}}} \frac{x_{\underline{k}} y_{\underline{\underline{h}}}}{\lambda_{\underline{k}}-\lambda_{\underline{h}}}\right| & =\left|\sum_{\substack{1 \leq u, v \leq v \\
u \neq v}} \frac{x_{i(u)} y_{i(v)}}{\lambda_{i(u)}-\lambda_{i(v)}}\right| \\
& \leq \frac{\pi}{\delta}\left(\sum_{1 \leq u \leq v}\left|x_{i(u)}\right|^{2}\right)^{1 / 2}\left(\sum_{1 \leq v \leq v}\left|y_{i(v)}\right|^{2}\right)^{1 / 2} \\
& =\frac{\pi}{\delta}\left(\sum_{\underline{k} \in E_{q}}\left|x_{\underline{k}}\right|^{2}\right)^{1 / 2}\left(\sum_{\underline{h} \in E_{q}}\left|y_{\underline{h}}\right|^{2}\right)^{1 / 2} .
\end{aligned}
$$

By applying Hilbert's inequality to each of the two sums of the right-term in (2.4) in parenthesis, we obtain

$$
\frac{(q !)^{2}}{T}\left|\sum_{\substack{\underline{k}, \underline{h} \in E_{q} \\ \underline{k} \neq \underline{\underline{k}}}} \frac{\mathbf{c}_{\underline{k}} \overline{\mathbf{c}}_{\underline{h}}}{\mathbf{l}_{\underline{k}}-\mathbf{l}_{\underline{h}}}-\sum_{\substack{\underline{k}, \underline{h} \in E_{q} \\ \underline{k} \neq \underline{\underline{h}}}} \frac{\mathbf{d}_{\underline{k}} \overline{\mathbf{d}}_{\underline{h}}}{\mathbf{l}_{\underline{k}}-\mathbf{l}_{\underline{h}}}\right| \leq \frac{2 \pi(q !)^{2}}{T \xi} \sum_{\underline{k} \in E_{q}}\left|\mathbf{d}_{\underline{k}}\right|^{2} \leq \frac{2 \pi q !}{T \xi} d(s, t)^{2 q},
$$

since

$$
\begin{aligned}
(q !)^{2} \sum_{\underline{k} \in E_{q}}\left|\mathbf{d}_{\underline{k}}\right|^{2} & =\sum_{k_{1}+\ldots+k_{N}=q}\left[\frac{q !}{k_{1} ! \ldots k_{N} !}\right]^{2} \prod_{n=1}^{N}\left|c_{n} \gamma_{n}\right|^{2 k_{n}} \\
& \leq q ! \sum_{k_{1}+\ldots+k_{N}=q} \frac{q !}{k_{1} ! \ldots k_{N} !} \prod_{n=1}^{N}\left|c_{n} \gamma_{n}\right|^{2 k_{n}}=q !\left[\sum_{n=1}^{N}\left|c_{n} \gamma_{n}\right|^{2}\right]^{q} \\
& =q !\left[\sum_{n=1}^{N}\left|c_{n}\right|^{2}\left|e^{i t \varphi_{n}}-e^{i s \varphi_{n}}\right|^{2}\right]^{q} \\
& =q !\left[4 \sum_{n=1}^{N}\left|c_{n}\right|^{2}\left|\sin \frac{(t-s) \varphi_{n}}{2}\right|^{2}\right]^{q}=q ! d(s, t)^{2 q}
\end{aligned}
$$

Similarly as before,

$$
\sum_{\underline{k} \in E_{q}}\left(\frac{q !}{k_{1} ! \ldots k_{N} !}\right)^{2} \prod_{n=1}^{N}\left|c_{n}\right|^{2 k_{n}}\left|e^{i t \varphi_{n}}-e^{i s \varphi_{n}}\right|^{2 k_{n}} \leq q !\left[\sum_{n=1}^{N}\left|c_{n} \gamma_{n}\right|^{2}\right]^{q}=q ! d(s, t)^{2 q}
$$

By substituting in (2.4), we therefore get

$$
\frac{1}{T} \int_{J}|P(\theta+t)-P(\theta+s)|^{2 q} d \theta \leq q !\left(1+\frac{2 \pi}{T \xi}\right) d(s, t)^{2 q} .
$$


Without Hilbert's inequality, it is possible to arrive to a similar result. We have with (2.2) and (2.8)

$$
\begin{aligned}
& \frac{1}{T} \int_{J}|P(\theta+t)-P(\theta+s)|^{2 q} d \theta \leq q ! d(s, t)^{2 q} \\
& +\sum_{\substack{k, h \in E_{q} \\
k \neq h}} \frac{(q !)^{2}}{k_{1} ! h_{1} ! \ldots k_{N} ! h_{N} !} \prod_{n=1}^{N}\left(c_{n} \gamma_{n}\right)^{k_{n}}\left(\overline{c_{n} \gamma_{n}}\right)^{h_{n}} \cdot\left|\frac{e^{i T \sum_{n=1}^{N}\left(k_{n}-h_{n}\right) \varphi_{n}}-1}{i T\left(\sum_{n=1}^{N}\left(k_{n}-h_{n}\right) \varphi_{n}\right)}\right| \\
& \leq q ! d(s, t)^{2 q}+\frac{2}{T \xi}\left(2 \sum_{n=1}^{N}\left|c_{n} \sin \frac{(t-s) \varphi_{n}}{2}\right|\right)^{q}\left(2 \sum_{n=1}^{N}\left|c_{n} \sin \frac{(t-s) \varphi_{n}}{2}\right|\right)^{q} \\
& =q ! d(s, t)^{2 q}+\frac{2}{T \xi}\left(2 \sum_{n=1}^{N}\left|c_{n} \sin \frac{(t-s) \varphi_{n}}{2}\right|\right)^{2 q} \\
& \leq\left(q !+\frac{2 N^{q}}{T \xi}\right) d(s, t)^{2 q},
\end{aligned}
$$

where we used the Cauchy-Schwarz inequality for getting the last estimate. Combining the two last estimates gives

$$
\frac{1}{T} \int_{J}|P(\theta+t)-P(\theta+s)|^{2 q} d \theta \leq\left(q !+\frac{2 \min \left(N^{q}, \pi q !\right)}{T \xi}\right) d(s, t)^{2 q} .
$$

Hence, the first in assertion (a). The same proof also yields mutatis mutandis

$$
\frac{1}{T} \int_{J}|P(\theta+s)|^{2 q} d \theta \leq\left(\sum_{n=1}^{N}\left|c_{n}\right|^{2}\right)^{q}\left(q !+\frac{2 \min \left(N^{q}, \pi q !\right)}{T \xi}\right)
$$

We start with

$$
P(\theta+t)^{q}=\left(\sum_{n=1}^{N} c_{n} e^{i \theta \varphi_{n}} e^{i t \varphi_{n}}\right)^{q}=\sum_{k \in E_{q}} \frac{q !}{k_{1} ! \ldots k_{N} !} \prod_{n=1}^{N} c_{n}^{k_{n}} e^{i \theta k_{n} \varphi_{n}} e^{i t \varphi_{n} k_{n}}
$$

and put this time $\gamma_{n}=e^{i t \varphi_{n}}$. Then all calculations made after (2.1) remain valid.

Proof of Corollary 2.2. The first assertion is immediate. As for the second, we have to estimate

$$
\xi_{\varphi}(N, q)=\inf _{\substack{\underline{h}, \underline{k} \in E_{q} \\ \underline{h} \neq \underline{\underline{k}}}}\left|\left(h_{1}-k_{1}\right) \varphi_{1}+\ldots+\left(h_{N}-k_{N}\right) \varphi_{N}\right|,
$$

when $\varphi_{n}=\log p_{n}$. Let $\underline{\ell}=\underline{h}-\underline{k}$ and put

$$
P^{+}=\prod_{\ell_{n}>0} p_{n}^{\ell_{n}}, \quad P^{-}=\prod_{\ell_{n}<0} p_{n}^{-\ell_{n}} .
$$

Let $M$ be defined by $p_{M} \leq N<p_{M+1}$. Note that $P^{+} \neq P^{-}$by assumption, and $\max \left(P^{+}, P^{-}\right) \leq p_{M}^{q}$. Suppose $P^{+}>P^{-}$. Then,

$$
\left|\ell_{1} \varphi_{1}+\ldots+\ell_{N} \varphi_{N}\right|=\left|\log \prod_{n=1}^{N} p_{n}^{\ell_{n}}\right|=\log \frac{P^{+}}{P^{-}} \geq \log \left(1+\frac{1}{P^{-}}\right) \geq \log \left(1+\frac{1}{p_{M}^{q}}\right) \geq \frac{1}{2 p_{M}^{q}}
$$


The case $P^{+}<P^{-}$is treated identically. Therefore,

$$
\xi_{\varphi}(N, q) \geq \frac{1}{2 p_{M}^{q}}
$$

And so it suffices to apply the first estimate to this case.

Proof of Theorem 2.3. Consider a covering of $L$ with intervals

$$
I_{j}=\left[\frac{j}{\tilde{\varphi}_{N}}, \frac{j+1}{\tilde{\varphi}_{N}}\right] \quad j=j_{1}, \ldots, j_{1}+H .
$$

Recall that $P_{\theta}(t)=\sum_{n=1}^{N} c_{n} e^{i(t+\theta) \varphi_{n}}$, and let

$$
Q_{\theta}(t)=i \sum_{n=1}^{N} c_{n} \varphi_{n} e^{i(t+\theta) \varphi_{n}}
$$

Then $\frac{d}{d t} P_{\theta}(t)=Q_{\theta}(t)$. Now using the elementary identity $f(\beta)-f(\alpha)=\int_{\alpha}^{\beta} f^{\prime}(t) d t$, for each $t \in I_{j}$, we have

$$
\left|P_{\theta}(t)\right| \leq\left|P_{\theta}\left(\frac{j}{\tilde{\varphi}}\right)\right|+\int_{0}^{1 / \tilde{\varphi}}\left|Q_{\theta}\left(\frac{j}{\tilde{\varphi}}+u\right)\right| d u .
$$

Moreover, using Hölder's inequality we have

$$
\sup _{t \in I_{j}}\left|P_{\theta}(t)\right|^{2 q} \leq c_{q}\left|P_{\theta}\left(\frac{j}{\tilde{\varphi}}\right)\right|^{2 q}+c_{q} \tilde{\varphi}^{1-2 q} \int_{0}^{1 / \tilde{\varphi}}\left|Q_{\theta}\left(\frac{j}{\tilde{\varphi}}+u\right)\right|^{2 q} d u .
$$

Then,

$$
\begin{aligned}
\sup _{t \in L}\left|P_{\theta}(t)\right|^{2 q} & \leq c_{q} \sup _{j_{1} \leq j \leq H}\left|P_{\theta}\left(\frac{j}{\tilde{\varphi}}\right)\right|^{2 q}+c_{q} \tilde{\varphi}^{1-2 q} \int_{0}^{1 / \tilde{\varphi}} \sup _{j_{1} \leq j \leq H}\left|Q_{\theta}\left(\frac{j}{\tilde{\varphi}}+u\right)\right|^{2 q} d u \\
& \leq c_{q} \sum_{j=j_{1}}^{H}\left|P_{\theta}\left(\frac{j}{\tilde{\varphi}}\right)\right|^{2 q}+c_{q} \tilde{\varphi}^{1-2 q} \int_{0}^{1 / \tilde{\varphi}} \sum_{j=j_{1}}^{H}\left|Q_{\theta}\left(\frac{j}{\tilde{\varphi}}+u\right)\right|^{2 q} d u,
\end{aligned}
$$

and so

$$
\sup _{t \in L}\left|P_{\theta}(t)\right|^{2 q} \leq c_{q} \sum_{j=j_{1}}^{H}\left|P_{\theta}\left(\frac{j}{\tilde{\varphi}}\right)\right|^{2 q}+c_{q} \tilde{\varphi}^{1-2 q} \int_{0}^{1 / \tilde{\varphi}} \sum_{j=j_{1}}^{H}\left|Q_{\theta}\left(\frac{j}{\tilde{\varphi}}+u\right)\right|^{2 q} d u .
$$

Now integrating with respect to $\theta$, and using Proposition 2.1 to bound each integral

$$
\int_{J}\left|P_{\theta}\left(\frac{j}{\tilde{\varphi}}\right)\right|^{2 q} d \theta, \quad \int_{J}\left|Q_{\theta}\left(\frac{j}{\tilde{\varphi}}+u\right)\right|^{2 q} d \theta
$$

gives the claimed result.

3. Proof of Theorem 1.1. The constants appearing in Turán's [3, 4] result (Section 1) are important. We have therefore explicited all constants appearing in our proof. 
We begin with applying Theorem 2.3 to

$$
P\left(N_{1}, N_{2}, t\right)=\sum_{N_{1} \leq p \leq N_{2}} p^{-i t}
$$

where $N \leq N_{1}<N_{2} \leq 2 N$. We have $\tilde{\varphi}_{N} \leq \sup \{\log p, p \leq 2 N\} \leq C \log N$, and by using (2.13),

$$
\mathcal{B} \leq\left(q !\left[1+\frac{2 \pi p_{N_{1}}^{q}}{|J|}\right]\right)^{1 / 2 q} \leq C_{q} \max \left(1, \frac{N^{q}}{|J|}\right)^{1 / 2 q} .
$$

Let $L$ be such that $|L| \geq 1$. Since $\pi(2 x)-\pi(x) \leq \frac{x}{\log x}$ for any integer $x>1$, we have $\pi\left(N_{2}\right)-\pi\left(N_{1}\right) \leq \pi(2 N)-\pi(N)<N / \log N$,

$$
\sum_{N \leq p \leq 2 N} \log ^{2} p \leq \log ^{2}(2 N) \sum_{N \leq p \leq 2 N} 1 \leq \frac{N \log ^{2}(2 N)}{\log N} \leq C N \log N .
$$

We get

$$
\begin{aligned}
\left\|\sup _{t \in L}\left|P .\left(N_{1}, N_{2}, t\right)\right|\right\|_{m_{J}, 2 q} \leq & C_{q} \max \left(1, \frac{N^{q}}{|J|}\right)^{1 / 2 q}(|L| \log N)^{1 / 2 q}\left\{\left(\frac{N}{\log N}\right)^{1 / 2}\right. \\
& \left.+\frac{1}{\log N}\left(\sum_{N \leq p \leq 2 N} \log ^{2} p\right)^{1 / 2}\right\} \\
\leq & C_{q}\left(\max \left(1, \frac{N^{q}}{|J|}\right)|L| \log N\right)^{1 / 2 q}\left(\frac{N}{\log N}\right)^{1 / 2}
\end{aligned}
$$

so that if $|J| \leq N^{q}$,

$$
\left\|\sup _{t \in L}\left|P .\left(N_{1}, N_{2}, t\right)\right|\right\|_{m_{J}, 2 q} \leq C_{q} \frac{N}{(\log N)^{1 / 2}}\left(\frac{|L| \log N}{|J|}\right)^{1 / 2 q} .
$$

The remaining part of the proof now consists of carefully adjusting the parameters to apply Turán's result (1.1).

Main parameters: $(\mathbf{H}, \delta, \mathbf{q}, \mathbf{B}, v, \mathbf{m}, \alpha)$. The constants $H, \delta, q, \alpha$ are numerical and fixed.

These will produce the constant $c$ in (1.1). See (3.14).

Let $H \geq 2$ be some integer. Put

$$
\delta=\frac{H-1}{8 H} \quad q=\frac{5}{1-8 \delta}=5 H .
$$

Then,

$$
0<\delta<1 / 8 \quad \text { and } \quad q>\frac{4(\delta+1)}{1-8 \delta}
$$

In addition, we set

$$
B=4 q \delta+2(\delta+1)
$$

and note that $2 B=8 q \delta+4(\delta+1)<q$. 
Now fix some positive integer $v$ and set

$$
U=2^{\nu}, \quad J=\left[U^{2 B}, 2 U^{2 B}\right], \quad L=\left[U^{B}, 8 U^{B}\right] .
$$

Let $N=2^{m}$ with $m \geq v$. It follows that $|J|=U^{2 B} \leq U^{q} \leq N^{q}$. Then,

$$
\left\|\sup _{2^{m} \leq N_{1}<N_{2} \leq 2^{m+2}} \sup _{t \in L}\left|P .\left(N_{1}, N_{2}, t\right)\right|\right\|_{m_{J}, 2 q} \leq C_{q} \frac{2^{m(1+1 / q)}}{m^{1 / 2}}\left(\frac{|L| m}{|J|}\right)^{1 / 2 q} .
$$

By Minkowski's inequality

$$
\begin{aligned}
& \left\|\sup _{\nu \leq m \leq \nu(1+\delta)} \sup _{2^{m} \leq N_{1}<N_{2} \leq 2^{m+2}} \sup _{t \in L}\left|P .\left(N_{1}, N_{2}, t\right)\right|\right\|_{m_{J}, 2 q} \\
\leq & \sum_{\nu \leq m \leq \nu(1+\delta)} \sup _{\nu 2^{m} \leq N_{1}<N_{2} \leq 2^{m+2}} \sup _{t \in L}\left|P .\left(N_{1}, N_{2}, t\right)\right| \|_{m_{J}, 2 q} \\
\leq & C_{q}\left(\frac{|L|}{|J|}\right)^{1 / 2 q} \sum_{\nu \leq m \leq \nu(1+\delta)} 2^{m(1+1 / q)} m^{1 / 2 q-1 / 2} \\
\leq & C_{q} v^{1 / 2 q-1 / 2}\left(\frac{|L|}{|J|}\right)^{1 / 2 q} \sum_{\nu \leq m \leq v(1+\delta)} 2^{m(1+1 / q)} \\
\leq & 2 C_{q} v^{1 / 2 q-1 / 2} 2^{-(B / 2 q) v} 2^{v(1+\delta)(1+1 / q)} .
\end{aligned}
$$

Now if $U \leq N \leq N_{1}<N_{2} \leq 2 N \leq U^{1+\delta}$, choose $v \leq m \leq v(1+\delta)$ such that $2^{m} \leq$ $N<2^{m+1}$. Then $2^{m} \leq N \leq N_{1}<N_{2} \leq 2 N<2^{m+2}$. Thus,

$$
\begin{aligned}
& \left\|\sup _{U \leq N \leq N_{1}<N_{2} \leq 2 N \leq U^{1+\delta}} \sup _{t \in L}\left|P .\left(N_{1}, N_{2}, t\right)\right|\right\|_{m_{J}, 2 q} \\
\leq & \left\|\sup _{\nu \leq m \leq \nu(1+\delta)} \sup _{2^{m} \leq N_{1}<N_{2} \leq 2^{m+2}} \sup _{t \in L}\left|P .\left(N_{1}, N_{2}, t\right)\right|\right\|_{m_{J}, 2 q} \\
\leq & 2 C_{q} 2^{\nu[(1+\delta)(1+1 / q)-(B / 2 q)]} v^{1 / 2 q-1 / 2} \\
\leq & 2 C_{q} 2^{[1-\delta] v} v^{1 / 2 q-1 / 2}:=M,
\end{aligned}
$$

since with our choices $(1+\delta)(1+1 / q)-B / 2 q=1-\delta$.

Next, let $0<\alpha<1$ be fixed and set $\mu(\alpha)=1 /(1-\alpha)^{1 /(2 q)}$. Set

$$
\tilde{J}=\left\{\theta \in J: \sup _{\substack{U \leq N \leq N_{1}<N_{2} \leq 2 N \leq U^{1+\delta} \\ \in \in L}}\left|P_{\theta}\left(N_{1}, N_{2}, t\right)\right| \leq \mu(\alpha) M\right\} .
$$

By the Tchebycheff inequality,

$$
\begin{aligned}
\frac{1}{|J|} \lambda\{J \backslash \tilde{J}\} & \leq \frac{1}{|J|(\mu M)^{2 q}} \int_{J} \sup _{\substack{U \leq N \leq N_{1}<N_{2} \leq 2 N \leq U^{1+\delta} \\
t \in L}}\left|P_{\theta}\left(N_{1}, N_{2}, t\right)\right|^{2 q} d \theta \\
& \leq \mu(\alpha)^{-2 q}=1-\alpha .
\end{aligned}
$$

Therefore, $\lambda\{\tilde{J}\} \geq \alpha|J|$ and for all $\theta \in \tilde{J}$,

$$
\sup _{\substack{U \leq N \leq N_{1}<N_{2} \leq 2 N \leq U^{1+\delta} \\ t \in L}}\left|P_{\theta}\left(N_{1}, N_{2}, t\right)\right| \leq 2 \mu(\alpha) C_{q} 2^{[1-\delta] v} v^{1 / 2 q-1 / 2} .
$$


Pick some $\theta$ in $\tilde{J}$. Then,

$$
\begin{aligned}
\sup _{\substack{U \leq N \leq N_{1}<N_{2} \leq 2 N \leq U^{1+\delta} \\
\tau \in \theta+L}}\left|\sum_{N_{1} \leq p \leq N_{2}} \frac{1}{p^{i \tau}}\right| & \leq 2 \mu(\alpha) C_{q} 2^{\nu(1-\delta)} v^{1 / 2 q-1 / 2} \\
& =2 \mu(\alpha) C_{q} U^{1-\delta}(\log U)^{1 / 2 q-1 / 2} \\
& \leq 2 \mu(\alpha) C_{q} \frac{U(\log U)^{1 / 2 q-1 / 2}}{U^{\delta}}
\end{aligned}
$$

But if $\tau \in \theta+L, \tau \leq 2 U^{2 B}+8 U^{B} \leq 3 U^{2 B}$ if $U$, namely $v$ is large enough. It follows that $U^{\delta} \geq C \tau^{\delta /(2 B)}$.

Put

$$
b:=\frac{\delta}{2 B}=\frac{\delta}{8 q \delta+4(\delta+1)} .
$$

We have obtained the following:

For all $\tau \in\left[\theta+U^{B}, \theta+8 U^{B}\right]$ and $U \leq N \leq N_{1}<N_{2} \leq 2 N \leq U^{1+\delta}$,

$$
\left|\sum_{N_{1} \leq p \leq N_{2}} \frac{1}{p^{i \tau}}\right| \leq 2 \mu(\alpha) C_{q} \frac{N(\log N)^{1 / 2 q-1 / 2}}{\tau^{b}} .
$$

A family of local zerofree regions: We use secondary parameters: $\delta_{0}, D, b$. Let

$$
T=T_{\theta}=\theta+3 \sqrt{\theta} .
$$

We may assume $\theta \geq 1$. On the one hand,

$$
T-\sqrt{T}=\theta+3 \sqrt{\theta}-\sqrt{\theta} \sqrt{1+3 / \sqrt{\theta}} \geq \theta+3 \sqrt{\theta}-2 \sqrt{\theta}=\theta+\sqrt{\theta} \geq \theta+U^{B} .
$$

And on the other, since $U^{2 B} \leq \theta \leq 2 U^{2 B}$,

$$
T+\sqrt{T}=\theta+3 \sqrt{\theta}+\sqrt{\theta} \sqrt{1+3 / \sqrt{\theta}} \leq \theta+5 \sqrt{\theta} \leq \theta+5 \sqrt{2} U^{B} \leq \theta+8 U^{B} .
$$

Hence, $[T-\sqrt{T}, T+\sqrt{T}] \subset \theta+L$ and estimate (3.9) is valid for $T-\sqrt{T} \leq \tau \leq T+$ $\sqrt{T}$. Further, as

$$
U^{2 B} \leq \theta \leq T=\theta+3 \sqrt{\theta} \leq 2 U^{2 B}+3 \sqrt{2} U^{B}=U^{2 B}\left[2+3 \sqrt{2} U^{-B}\right] \leq 7 U^{2 B},
$$

it is also valid in the restricted range of values

$$
T^{\frac{1}{2 B}} \leq N \leq N_{1}<N_{2} \leq 2 N \leq\left(\frac{T}{7}\right)^{\frac{1+\delta}{2 B}}
$$

Now select a positive real $\delta_{0}$ such that

$$
0<\frac{2 \delta_{0}}{1-\delta_{0}}<\delta
$$


We note that $1+\delta-\frac{1+\delta_{0}}{1-\delta_{0}}=\delta-\frac{2 \delta_{0}}{1-\delta_{0}}>0$. Choose $v$ sufficiently large so that $2^{v\left[\delta-\frac{2 \delta_{0}}{1-\delta_{0}}\right]} \geq 7^{1+\delta}$. Since $2 B>1$, we have

$$
T^{1+\delta-\frac{1+\delta_{0}}{1-\delta_{0}}} \geq 2^{2 B \nu\left(1+\delta-\frac{1+\delta_{0}}{1-\delta_{0}}\right)} \geq 2^{\nu\left[1+\delta-\frac{1+\delta_{0}}{1-\delta_{0}}\right]}=2^{\nu\left[\delta-\frac{2 \delta_{0}}{1-\delta_{0}}\right]} \geq 7^{1+\delta},
$$

namely

$$
\left(\frac{T}{7}\right)^{1+\delta} \geq T^{\frac{1+\delta_{0}}{1-\delta_{0}}}
$$

Next, put

$$
D=\frac{1}{2 B\left(1-\delta_{0}\right)}
$$

Then (3.10) implies the admissibility of the more suitable field of parameters

$$
T^{D\left(1-\delta_{0}\right)}=T^{\frac{1}{2 B}} \leq N \leq N_{1}<N_{2} \leq 2 N \leq T^{D\left(1+\delta_{0}\right)}=T^{\frac{1+\delta_{0}}{2 B\left(1-\delta_{0}\right)}} \leq\left(\frac{T}{7}\right)^{\frac{1+\delta}{2 B}} .
$$

Estimate (3.9) then implies

$$
\left|\sum_{N_{1} \leq p \leq N_{2}} \frac{1}{p^{i \tau}}\right| \leq 2 \mu(\alpha) C_{q} \frac{N(\log N)^{1 / 2 q-1 / 2}}{\tau^{b}}
$$

for all $\tau \in\left[T-T^{1 / 2}, T+T^{1 / 2}\right]$ and all $T^{D\left(1-\delta_{0}\right)} \leq N \leq N_{1}<N_{2} \leq 2 N \leq T^{D\left(1+\delta_{0}\right)}$.

Recall that $0<\delta<1 / 8$ and $q=\frac{5}{1-8 \delta}$. Thus,

$$
B=4 q \delta+2(\delta+1)<\frac{20 \delta}{1-8 \delta}+\frac{9}{4}=\frac{80 \delta+9-72 \delta}{4(1-8 \delta)}=\frac{8 \delta+9}{4(1-8 \delta)}<\frac{5}{2(1-8 \delta)},
$$

and

$$
b=\frac{\delta}{2 B} \geq \frac{\delta(1-8 \delta)}{5} .
$$

In order that $b^{1 / 6} \geq \delta_{0}$, it suffices that $\frac{\delta(1-8 \delta)}{5} \geq(\delta / 2)^{6}$, namely $1-8 \delta \geq\left(5 / 2^{6}\right) \delta^{5}$, which is fulfilled if $\delta<1 / 9$, for instance, namely recalling that $\delta=\frac{H-1}{8 H}$ if $H<9$, which we do.

Thus, $b \geq \delta_{0}^{6}$ does hold, and (3.12) implies that the inequality

$$
\left|\sum_{N_{1} \leq p \leq N_{2}} \frac{1}{p^{i \tau}}\right| \leq c \frac{N(\log N)^{1 / 2 q-1 / 2}}{\tau^{\delta_{0}^{6}}}
$$

with (recalling that $\left.\mu(\alpha)=1 /(1-\alpha)^{1 /(2 q)}\right)$

$$
c=2 \mu(\alpha) C_{q},
$$

holds for all $\tau \in\left[T-T^{1 / 2}, T+T^{1 / 2}\right]$ and all $T^{D\left(1-\delta_{0}\right)} \leq N \leq N_{1}<N_{2} \leq 2 N \leq$ $T^{D\left(1+\delta_{0}\right)}$. 
Turán's $[3,4]$ result (Section 1) then implies that

$$
\zeta(\sigma+i t) \neq 0, \quad \forall \sigma>1-\delta_{0}^{12}, \quad \forall t \in\left[T_{\theta}-T_{\theta}^{1 / 2}, T_{\theta}+T_{\theta}^{1 / 2}\right] .
$$

But this holds for any $\theta \in \tilde{J}$ (recalling that $\lambda(\tilde{J}) \geq \alpha|J|, J=\left[2^{2 B \nu}, 2^{2 B \nu+1}\right]$ ), and for any $\nu$, assuming this one is large enough, depending on $\delta$, say $v_{\delta}$. We also recall that $\delta$ was fixed from the beginning (see 'Main parameters').

REMARK 3.1. Finding one $\theta$ in $J$ such that $\zeta(\sigma+i t) \neq 0$ for all $t$ in $\left[T_{\theta}-T_{\theta}^{1 / 2}, T_{\theta}+\right.$ $\left.T_{\theta}^{1 / 2}\right]$ and $\sigma>\sigma_{0}$, for some $\sigma_{0}<1$, can be deduced from Carlson's estimate on the number of zeros of the Riemann zeta function. The point here is that we have a measurable set of values of $\theta$ 's of measure close to the one of $J$, for which this is valid. This together with a simple covering argument will permit to exhibit a much bigger zerofree zone.

A semi-global zerofree region: Let $\psi(\theta)=\theta+3 \sqrt{\theta}$. The indice $v$ with $v \geq v_{\delta}$ being now temporarily fixed, let $\left.J_{0}=\right] 2^{2 B v}, 2^{2 B v+1}[\backslash \tilde{J}$. Using the fact that $\lambda(\psi([a, b]))=(b-a)+$ $3(\sqrt{b}-\sqrt{a}) \leq(b-a)\left\{1+2.2^{-B \nu}\right\}$, one can show

$$
\lambda\left(\psi\left(J_{0}\right)\right) \leq\left\{1+1 / 2^{B \nu}\right\}(1-\alpha) \lambda(J) .
$$

Let $\eta>0, J_{0}$ being an open set, $J_{0}=\cup_{n=1}^{\infty} I_{n}$, where $I_{n}$ are open intervals. Let $U_{N}=$ $\cup_{n=1}^{N} I_{n}$. Writing $U=U_{N} \bullet B$ with $B \subset \cup_{n=N+1}^{\infty} I_{n}$, we have

$$
\begin{aligned}
\lambda\left(\psi\left(J_{0}\right)\right) & \leq \lambda\left(\psi\left(U_{N}\right) \cup \psi(B)\right) \leq \lambda\left(\psi\left(U_{N}\right)\right)+\sum_{n=N+1}^{\infty} \lambda\left(\psi\left(I_{n}\right)\right) \\
& \leq \lambda\left(\psi\left(U_{N}\right)\right)+\left\{1+2.2^{-B \nu}\right\} \sum_{n=N+1}^{\infty} \lambda\left(I_{n}\right) \leq \lambda\left(\psi\left(U_{N}\right)\right)+\eta\left\{1+2.2^{-B \nu}\right\},
\end{aligned}
$$

assuming $N$ is large enough. Further, $\cup_{n=1}^{N} I_{n}=\cup_{n=1}^{N^{\prime}} I_{n}^{\prime}, I_{n}^{\prime}$ being pairwise disjoint intervals. Since $\psi$ is continuous increasing,

$$
\begin{aligned}
\lambda\left(\psi\left(U_{N}\right)\right) & =\lambda\left(\sum_{n=1}^{N^{\prime}} \psi\left(I_{n}^{\prime}\right)\right)=\sum_{n=1}^{N^{\prime}} \lambda\left(\psi\left(I_{n}^{\prime}\right)\right) \leq\left\{1+2.2^{-B \nu}\right\} \sum_{n=1}^{N} \lambda\left(I_{n}\right) \\
& =\left\{1+2.2^{-B \nu}\right\} \lambda\left(U_{N}\right) \leq\left\{1+2.2^{-B \nu}\right\}\left(\lambda\left(J_{0}\right)+\eta\right) .
\end{aligned}
$$

Thus,

$$
\lambda\left(\psi\left(J_{0}\right)\right) \leq\left\{1+1 / 2^{B \nu}\right\} \lambda\left(J_{0}\right)+2 \eta\left\{1+1 / 2^{B \nu}\right\} \leq\left\{1+1 / 2^{B \nu}\right\}\{(1-\alpha) \lambda(J)+2 \eta\},
$$

since $\lambda\left(J_{0}\right) \leq(1-\alpha) \lambda(J)$. Since $\eta$ is arbitrary, (3.16) follows.

Therefore,

$$
\begin{aligned}
\lambda(\psi(\tilde{J})) & \geq \lambda(\psi(J))-\left\{1+2^{-B \nu}\right\}(1-\alpha) \lambda(J) \\
& =\lambda(\psi(J))\left[1-\frac{1+2^{-B \nu}}{1+3(\sqrt{2}-1) 2^{-B \nu}}(1-\alpha)\right] \\
& :=(1-\bar{\alpha}) \lambda(\psi(J)),
\end{aligned}
$$

noting that $\lambda(\psi(J))=\lambda(J)\left(1+3(\sqrt{2}-1) 2^{-B v}\right)$. 
As $T_{\theta}^{1 / 2} \geq \theta^{1 / 2} \geq 2^{B \nu}$, we have $\left[T_{\theta}-T_{\theta}^{1 / 2}, T_{\theta}+T_{\theta}^{1 / 2}\right] \supset\left[T_{\theta}-2^{B \nu}, T_{\theta}+2^{B \nu}\right]$. Now consider on $\psi(J)=\left[\psi\left(2^{2 B v}\right), \psi\left(2^{2 B v+1}\right)\right]$ the subdivision

$$
K_{i}=\left[\psi\left(2^{2 B \nu}\right)+(i-1) 2^{B \nu-1}, \psi\left(2^{2 B \nu}\right)+i 2^{B \nu-1}\right], \quad 1 \leq i \leq\left(2^{B \nu+1}+6(\sqrt{2}-1)\right) .
$$

In view of (3.17), the number of indices $i$ such that $\left.K_{i} \cap \psi(\tilde{J})\right)=\emptyset$ is less than $(1-$ $\bar{\alpha}) \lambda(\psi(J)) / 2^{B v+1}$.

Consequently, at least $\bar{\alpha} \lambda(\psi(J)) / 2^{B v+1}$ indices $i$ are such that $\left.K_{i} \cap \psi(\tilde{J})\right) \neq \varnothing$. Pick a real $\vartheta$ in the intersection. We have

$$
\left[\vartheta-\vartheta^{1 / 2}, \vartheta+\vartheta^{1 / 2}\right] \supset K_{i},
$$

so that by (3.15),

$$
\zeta(\sigma+i t) \neq 0, \quad \forall \sigma>1-\delta_{0}^{12}, \quad \forall t \in K_{i},
$$

and the number of indices $i$ for which this is true, exceeds

$$
\bar{\alpha} \lambda(\psi(J)) / 2^{B \nu+1}=\bar{\alpha}\left(2^{B \nu+1}+6(\sqrt{2}-1)\right) .
$$

We can now achieve the proof. Given any fixed real $0<\alpha^{*}<1$, it follows from (3.18) and (3.19) that in any subdivision of $\psi(J)$ of size $2^{B v-1}$, at least $\alpha^{*} 2^{B v+1}$ intervals are zerofree. Since $\psi(J)=\left[2^{2 B v}+3.2^{B v}, 2.2^{2 B v}+3 \sqrt{2} .2^{B v}\right]$, it also implies that in any subdivision of $\left[2^{2 B \nu}, 2^{2 B \nu+1[}\right.$ of size $2^{B \nu-1}$, at least $\alpha^{*} 2^{B \nu+1}$ intervals are zerofree.

ACKNOWLEDGEMENT. We wish to thank the referee who carefully read the paper, and suggested another more direct way to prove Theorem 2.3 than the one we initially gave to control suprema.

\section{REFERENCES} 1927).

1. E. Landau, Vorlesungen über Zahlentheorie, vol. I-II (S. Hirzel, Leipzig, Germany,

2. H. Montgomery, Ten lectures on the interface between analytic number theory and harmonic analysis, Regional Conference Series in Math., vol. 84 (Conference Board of the Math. Sciences, Washington DC, 1993).

3. P. Turán, Eine neue Methode inter Analysis und deren Anwendungen (Akadémiai Kiadó, Budapest, Hungary, 1953).

4. P. Turán, Exponential sums and the Riemann conjecture, in Analytic number theory, Proc. Symp. Pure Math. vol. XXIV, St. Louis Univ., St Louis, MO, 1972 (Amer. Math. Soc. Providence, RI, 1973), 305-314. 